formation of collagen. Prolidase catalyses the hydrolysis of oligo/dipeptides which contains proline or hydroxyproline in the carboxyl terminal position. Many studies have identified prolidase activity at varying levels in fibrotic diseases. The aim of the study is to investigate the relationship between silicosis and prolidase activity.

Methods This study is a cross-sectional study. We reviewed medical records (functional status and radiological findings) and serum prolidase levels of 62 patients who were admitted to our centre between September and December 2015.

Result All cases were male and silicosis was diagnosed in 37 (\%) of 62 cases. Prolidase level was significantly higher in patients with silicosis than in normal $(\mathrm{p}<0.001) \quad(4877$ \pm 2324 vs $815 \pm 520$ ). Correlation analysis showed significant positive correlation between prolidase activity and ILO classification ( $\mathrm{r}: 0,723, \mathrm{p}<0,001$, figure $1 \mathrm{~A})$ and age ( $\mathrm{r}: 0,391$, $\mathrm{p}: 0,002)$. Prolidase activity was negatively correlated with FEV1 (r:-0.332, p: 0.008) and FCV (r:-0.295, p:0.020).

Discussion Since prolidase is involved in collagen metabolism, its activity can be used for early diagnosis and follow-up of silicosis. Further studies are needed to clarify the etiopathogenetic mechanisms of silicotic nodule formation and early detection of vulnerable population who had silica exposure before nodule formation.

\section{PNEUMOCONIOSIS AMONG DENTAL TECHNICIANS}

Nur Şafak Alici, Ayşe Coşkun Beyan, Yücel Demiral, Arif Çimrin. Department of Occupational Health and Diseases, Faculty of Medicine, Dokuz Eylul University, Izmir, Turkey

\subsection{6/oemed-2018-ICOHabstracts. 1286}

Introduction Dental technicians are exposed to various chemicals, including silica particles and metals. The aim of this study is to explore the pneumoconiosis risk among dental technicians.

Methods This is a cross-sectional study. We reviewed medical records of patients diagnosed with pneumoconiosis who were admitted to outpatient occupational disease centre between 2013 and 2015. Pneumoconiosis was diagnosed by X-ray radiograms in accordance to ILO procedures and with High Resolution Computarized Tomography (HRCT).

Result Pneumoconiosis was diagnosed in 46 (65.7\%) of the 70 dental technicians who were referred to our hospital. There were $45(97.8 \%)$ male and 1 (2.2\%) female cases. Radiologically, 16 cases were defined as $2 / 3$ or more of profusion and 11 cases had large opacity. In $3(6,5 \%)$ cases who had profusion $0 / 1$, The most frequent findings are micronodules and lymphadenopathy in HRCT. Consolidation, conglomerate masses and ground glass opacities are also described alongside the reticular opacities. There was a poor correlation between pulmonary function tests and profusion, (correlation coefficient were between: $-0,18$ and $-0,058$ ). There was no correlation observed between profusion and age started to work and exposure duration.

Discussion The study showed that pneumoconiosis among dental technicians is a great risk. Especially sandblasting procedures is raise the risk of pneumoconiosis. This shows that there are serious limitations in control measures and employee' health monitoring.
996

RETROSPECTIVE STUDY OF THE PULMONARY FINDINGS IN COMPLEMENTARY EXAMS IN RETIRED MINING WORKERS EXPOSED TO ASBESTOS

LFT Priester*, VL Ferreira, LR Ferreira, RCG Bianchi, CM Galhardi. Centro Universitário das Faculdades Associadas de Ensino, São João da Boa Vista, Brazil

10.1136/oemed-2018-ICOHabstracts. 1287

Introduction Asbestos, a silicatum largely used in industry is responsible to harmful effects to the respiratory system, once inhalation and fibre accumulation of these materials in the pulmonary parenchyma are associated to pleural and pulmonary injuries. The objective of this study is to find the most prevalent pulmonary changes evaluated in asbesto's exposed workers and verify if there is any risk factor associated.

Methods This was a cross-sectional study which evaluated 48 retired workers exposed more than 15 years to asbestos at aluminium mining industry at Poços de Caldas - Brazil. The workers were evaluated between June 2015-July 2016 and submitted to a structured analysis for data collection including: gender, age, smoking load, chest X-ray, chest computed tomography (CT), and spirometry (divided in normal, mild, moderate and severe alterations). The CT was applied in workers who had shown clinic, radiographic and spirometric alterations. It was applied Chi-Square method, $\mathrm{p}<0,05$ using One-Way ANOVA and Tukey test.

Results All workers were male, mean age of 64 years-old. The mean FEV1 was higher in non-smokers group (95\%) in comparison to ex-smokers group (88\%) and smokers (82\%). The FEV1 decay was proportional to smoking load increase; FVC lowest values were found in the smokers group (mean of $78 \%$ ) and increasing FVC values in ex-smokers and non-smokers group. Lung emphysema and diffuse bronchial injury were the most prevalent findings, shown on 8 and 7 workers respectively. Only 2 workers presented pleural plaques.

Discussion The results suggested that smoking is an important risk factor for functional lung injuries, enhancing the harmful effects of asbestos chronic exposure. These data may contribute with strategies to enhance smoking cessation and preventive respiratory disease programs within the mining industry in order to prevent pulmonary injuries. These measures may possibly decrease the risk for developing lung diseases associated in asbestos' exposed mining workers.

\section{UNDER-REPORTED ASBESTOS-RELATED LUNG CANCER IN TAIWAN}

${ }^{1}$ Heng-Hao Chang* ${ }^{1,2}$ Chen-Long Wu, ${ }^{1}$ Jung-Der Wang, ${ }^{1}$ Yau-Chang Kuo. ${ }^{1}$ Department of Occupational and Environmental Medicine, National Cheng Kung University Hospital, Tainan, Taiwan; ${ }^{2}$ Center for Prevention and Treatment of Occupational Injury and Disease, Southern Taiwan, Tainan, Taiwan

\subsection{6/oemed-2018-ICOHabstracts. 1288}

Introduction Mesothelioma is a rare malignancy, primarily caused by exposure to asbestos. The incidence rate of mesothelioma in Taiwan increased steadily in the previous two decades. However, very few of the mesothelioma patients received compensation due to their asbestos-related work. This study aims to examine the status of reported and unreported asbestos-related lung cancer, particularly mesothelioma in 
Taiwan, and to provide recommendations for improving the surveillance system.

Methods The reporting system of occupational diseases initiated by Department of Labours was used to retrieve the cases of occupational lung cancer from 2008 to 2014. Descriptive analysis was conducted including identification of exposure to asbestos. We further compared the data with Taiwan Cancer Registry. A review for international comparison of mesothelioma surveillance system was performed.

Results 73 cases of occupational lung cancer were reported, and 42 were suspected to be asbestos-related. 31 cases were confirmed as malignant mesothelioma. Only one of the 42 asbestos-related lung cancer cases was female. Their occupations and industries included construction (36\%), work concerning installation and repair of boilers (24\%), and shipyard and ship breaking (19\%). The year of age at the time of diagnosis is 60.5 , while the induction time was 35.2 years. In the same period, 349 mesothelioma cases were identified in the Taiwan Cancer Registry.

Discussion This study showed that very few mesothelioma patients seek compensation in Taiwan. Further review showed that mesothelioma surveillance system was established in many countries to provide information of mesothelioma epidemic and investigate in asbestos exposure. Some have a specific registry and rely on medical doctor, particularly pathologists, to report. Some directly link the data from the pre-existing cancer registry. In Taiwan, all hospitals were mandated to submit cancer data to the central cancer registry. Improving linkage between mesothelioma surveillance and cancer registry should be considered.

\section{ACCELERATED PNEUMOCONIOSIS BY ALGINATES IN A WORKER IN THE CHEMICAL-PHARMACEUTICAL INDUSTRY. CASE REPORT}

E Frias*, M Valdes. UIDAC, Research Unit, Teaching and Clinical Support in Occupational Health, IMSS, Guadalajara, Mexico

\subsection{6/oemed-2018-ICOHabstracts. 1289}

Introduction Pneumoconiosis is a respiratory disease produced by the inhalation of silicon dioxide, in its crystalline and cryptocrystalline forms, it is common to accompany other powders in the working environment. The accelerated form develops within the first ten years of the beginning of exposure to high silica level, presenting symptoms consisting of chronic cough and dyspnea of exertion accompanied by worsening of radiological images; with a national rate in Mexico of $0.48-$ 2.39 cases per 10000 workers.

Methods 27-year-old female worker, production manager and responsible for the area of alginates in a manufacturing company for the dental industry for 6 years, with inhalation exposure to inorganic powders and alginate vapours whose composition is calcined diatomaceous flow. Begins current disease after entering the alginate area, presenting dry cough, burning sensation in the nose and epistaxis, without going to medical attention. 5 years later she presented dyspnea of great efforts and non-productive cough, evolving at 5 months to dyspnea of moderate efforts and tachycardia; with spirometry that concluded severe restriction. Pneumoconiosis was diagnosed 10 months later by Pneumology, presenting severe dyspnea, tachycardia and nail cyanosis at moderate efforts, requiring the use of home and ambulatory oxygen and treatment with bronchodilators.

Results Symmetrical thorax, decreased respiratory movements, fine rales in the right hemithorax and upper lobe of the left hemithorax, Plethysmography FVC 47\%, TLC 64.4\%, sixminute walk test suspended at 2 min by $\mathrm{SO} 2<85 \%$, oscillometry: increased resistance in distal airway, bronchoscopy with cytopathologic: negative to malignancy, BAAR (-).

Discussion High exposure and no personal protective equipment suitable for alginates within industrial processes can lead to the accelerated development of pneumoconiosis; in the company visit, the inhalation exposure to composite powders with calcined diatomaceous flow was corroborated, which together with the paraclinical studies and the symptomatology presented demonstrated the cause-effect relationship, workinjury.

\section{EARLY DETECTION OF ASBESTOS-RELATED LUNG CANCER BY LOW-DOSE MULTISLICE-CT (LOW-DOSE MSCT)}

V Harth*. Institute for Occupational Medicine and Maritime Medicine (ZAM), University Medical Centre Hamburg-Eppendorf, Hamburg, Germany

\subsection{6/oemed-2018-ICOHabstracts. 1290}

Introduction Lung cancer is the most common cause of death from cancer worldwide, estimated to be responsible for nearly one in five (18\%), or 1.38 million, cancer deaths in 2008. Of all risk factors, smoking has been identified as the major risk factor. Other causes of lung cancer include occupational (e.g. asbestos) and environmental exposures (e.g. radon decay products). Despite the reduction or ban of asbestos use in many countries, the global incidence of asbestos-related lung cancer is still increasing. Nevertheless, asbestos is still produced and exported in some countries in the world. The National Lung Screening Trial (NLST) enrolled persons at high risk for lung cancer to undergo annual screenings with either low-dose CT or single-view posteroanterior chest radiography. In the low CT-group, mortality from lung cancer was reduced by $20.0 \%$. Currently, secondary prevention strategies are extensively discussed to reduce mortality from lung cancer.

Methods In Germany, more than $80 \%$ of lung cancers are diagnosed at an advanced disease stage (clinical stages IIIa, IIIb, and IV) where the survival rate is poor. Since lung cancer is only curable at an early stage of the disease, in Germany, formerly asbestos-exposed insured individuals have the statutory right to receive 'follow-up occupational medical examinations' which target the early detection of asbestosrelated diseases. Recently, the German Social Accident Insurance (DGUV) founded a working group to establish an annual low-dose MSCT scanning program.

Results The eligibility criteria for participants are: at least 10 years of exposure to asbestos (starting before 1985) or a recognised case of asbestos-induced occupational disease (No. 4103 BKV), between 55 and 74 years of age and a history of cigarette smoking of at least 30 pack years. The participants are contacted by GVS (a joint organisation involving all German social accident insurance institutions) or the specific statutory accident insurance and examinations are offered which are carried out locally by selected physicians. A quit-smoking counselling is provided, and participants are asked to donate blood for biomarker research. For MSCT scanning, at least 16 